\title{
The H3ABioNet helpdesk: an online bioinformatics resource, enhancing Africa's capacity for genomics research
}

\author{
Judit Kumuthini ${ }^{*}$, Lyndon Zass ${ }^{1}$, Sumir Panji ${ }^{2}$, Samson P. Salifu ${ }^{3}$, Jonathan K. Kayondo ${ }^{4}$, Victoria Nembaware ${ }^{2}$, \\ Mamana Mbiyavanga ${ }^{2}$, Ajayi Olabode ${ }^{1}$, Ali Kishk ${ }^{5}$, Gordon Wells ${ }^{1}$, Nicola J. Mulder ${ }^{2}$ and as members of the \\ Sustainability and Outreach Work Package of the H3ABioNet Consortium
}

\begin{abstract}
Background: Currently, formal mechanisms for bioinformatics support are limited. The H3Africa Bioinformatics Network has implemented a public and freely available Helpdesk (HD), which provides generic bioinformatics support to researchers through an online ticketing platform. The following article reports on the H3ABioNet HD (H3A-HD)'s development, outlining its design, management, usage and evaluation framework, as well as the lessons learned through implementation.

Results: The H3A-HD evaluated using automatically generated usage logs, user feedback and qualitative ticket evaluation. Evaluation revealed that communication methods, ticketing strategies and the technical platforms used are some of the primary factors which may influence the effectivity of HD.

Conclusion: To continuously improve the H3A-HD services, the resource should be regularly monitored and evaluated. The H3A-HD design, implementation and evaluation framework could be easily adapted for use by interested stakeholders within the Bioinformatics community and beyond.
\end{abstract}

Keywords: Bioinformatics support, Genomics support, H3Africa, H3ABioNet, Helpdesk

\section{Background}

In the last decade, the ability to perform bioinformatics analyses has become crucial to biomedical science [1]. Due to collaborative efforts and increased funding across Africa, the continent has steadily developed its bioinformatics capacity over recent years [2, 3]. H3ABioNet (H3Africa Bioinformatics Network, www.h3abionet.org) is a pan-African bioinformatics network which supports such development $[4,5]$. The network has successfully built capacity through various strategies, including, upgrading computing infrastructure in Africa; developing and customizing software tools to facilitate data management and manipulation; initiating collaborative multidisciplinary research partnerships,

\footnotetext{
*Correspondence: jkumuthini@gmail.com

A full list of H3ABioNet consortium members can be found at www.h3 abionet.org/home/consortium.

${ }^{1}$ Centre for Proteomic and Genomic Research, Cape Town, Western Cape, South Africa

Full list of author information is available at the end of the article
}

and providing blended bioinformatics learning for researchers across the continent [4-6].

Generic bioinformatics support is typically achieved through research collaboration, consultancy, and, most frequently, informal online communications $[7,8]$. To our knowledge, few formal or professional mechanisms of generic bioinformatics support currently exist. Helpdesks (HDs) are commonly employed as core support resources, both academically and commercially, and function as important channels which aid the communication of complex concepts [9]. However, few bioinformatics HDs have been established, and these are often resource-, community- or region-specific [10, 11].

Due to the lack of established bioinformatics support resources available to African researchers, H3ABioNet established an online bioinformatics HD (henceforth referred to as the H3A-HD) to aid bioinformatics capacity development. To our knowledge, the H3A-HD is one of the first public and freely available, generic bioinformatics

(C) The Author(s). 2019 Open Access This article is distributed under the terms of the Creative Commons Attribution 4.0 International License (http://creativecommons.org/licenses/by/4.0/), which permits unrestricted use, distribution, and 
HDs, systematically implemented to provide rapid bioinformatics support across Africa and the rest of the world. The following report introduces the H3ABioNet bioinformatics HD, and outlining its function and use, and providing suggestions for future HD implementations.

\section{The H3ABioNet helpdesk (H3A-HD)}

The H3A-HD provides bioinformatics and H3ABioNetspecific project support through technical experts (or H3AHD representatives), with backgrounds in various bioinformatics sub-disciplines [3]. Representatives are H3ABioNet members maintained on a voluntary basis, ranging from midto late-career scientists, and selected based on their qualifications and research expertise. Based on the overlap between expertise and query categories, an H3A-HD administrator prioritizes and categorizes queries as simple, intermediate or advanced, before assigning them to the HD representative of the relevant query category. A given representative handles a single query category; and if an assigned query remains unresolved, the query is reassigned as necessary and the ticket history is shared across HD representatives.

A technical platform for the H3A-HD was selected on the basis of being cost free, easy to maintain and use, as well as being customizable to the existing H3ABioNet website. The tool should also allow an unlimited number of ticket categories, with an unlimited number of representatives. Therefore, an open-source tool built using Django, django-helpdesk, was selected for the front- and backend of the H3A-HD. Designed for small businesses to receive, manage and respond to requests for help from external users, or other people within your institute. Representatives can view the ticket dashboard containing assigned and unassigned tickets, as well as the basic status of the helpdesk. They are able to search through all tickets, save their searches for future use, and follow up or respond to tickets.

The H3A-HD accepts queries, submitted as tickets, through an online interface at https://helpdesk.h3abionet. org/. Users are encouraged to create logins to aid H3A-HD maintenance. An administrator assigns tickets to representatives, who resolve and close tickets. The H3A-HD supports a wide range of query categories. These categories are broadly arranged into 3 overall category types; Analysis categories pertaining to the analysis of genetic data; $\mathrm{H} 3 \mathrm{ABi}-$ oNet Tools and Services - dedicated to H3ABioNet associated projects and outputs; and Additional Support geared to general technical support. The H3A-HD thus differs from other support platforms due to the type and level of support provided. Users can also browse and access additional resources which complement the HD services, including standard operating procedures (SOPs), workflows and pipelines for Analysis categories, and support guidelines and material for H3ABioNet Tools and Services, and Additional Support categories (Table 1).

\section{Results}

The H3A-HD is regularly monitored and evaluated using built-in Joomla functionality, qualitative analysis and user feedback.

\section{Usage and query resolution time}

Based on the automatically generated logs, 320 queries were submitted to the H3A-HD between 1 August 2012 and 1 August 2017. Figure 1 illustrates the query volumes, average resolution times and the number of responding $\mathrm{HD}$ representatives, during this period. The left axis illustrates the distribution of query volume and average resolution time (in minutes), and the right axis illustrates number of responding H3A-HD representatives, over time.

There was an overall increase in H3A-HD usage over time, with peak usage between 2016 and 2017. Notably, despite increased query volume and no change in the amount of responding $\mathrm{HD}$ representatives over time, there was an overall decrease in query resolution time. This indicates the HD representatives' improved capacity and query knowledge over time.

\section{Feedback}

Feedback from previous H3A-HD users and potential network users was determined using an online survey, distributed to 244 participants. Of these participants, 128 (53\%) responded; the majority $(75 \%)$ had not previously used the H3A-HD, stating 'not yet required' (53\%) and 'lack of awareness' (24\%) as the primary reasons. Others (19\%) expressed a need for category expansion, requesting cytogenetics, metagenomics, deep learning etc. The remaining respondents (25\%) had previously used the H3A-HD. The majority of respondents had mixed research backgrounds (26\%), followed closely by genetics (23\%) (Fig. 2).

As illustrated in Fig. 3, H3A-HD user satisfaction was measured on a standard 1-5 scale (very poor, poor, average, good, very good), assessing the quality of support received, resolution time and user friendliness of the H3A-HD interface. The majority of H3A-HD users rated quality of support (77\%), query resolution time (74\%), and user friendliness of the H3A-HD interface (84\%), average and above.

Figure 4 summarizes H3A-HD users' answers to a series of closed questions. Responses were generally positive and only $6 \%$ of previous users responded that they wouldn't use or recommend the use of the H3AHD in the future. In addition, only $30 \%$ of HD users' queries were not resolved. These users were generally preferred more interactive communication, as they did not know who to follow up with regarding delayed response.

\section{Qualitative analysis}

To identify recurrent themes and concerns, queries were qualitatively analysed on a ticket-by-ticket basis. Recurrent 
Table 1 Query Categories and Standard Operating Protocols supported by the Help Desk

\begin{tabular}{|c|c|c|}
\hline Overall Category Type & Query Category & Associated Workflows \\
\hline \multirow[t]{8}{*}{ Analysis } & Analysis - NGS & Human Variant Calling Workflow \\
\hline & Analysis - GWAS & Variant Discovery and Prioritisation \\
\hline & H3Africa Genotyping Array & GWAS Pipeline \\
\hline & Microbiome Analysis & RNA-seq Workflow \\
\hline & Chip Imputation & 16S rRNA Diversity Analysis Workflow \\
\hline & Variant Calling Analysis & \multirow[t]{3}{*}{ Imputation Pipeline } \\
\hline & Machine Learning & \\
\hline & Biostatistics & \\
\hline \multirow[t]{8}{*}{ H3ABioNet Tools \& Services } & Standard CRF & \multirow{8}{*}{$\begin{array}{l}\text { Associated information packets, guidelines, } \\
\text { and tools. }\end{array}$} \\
\hline & Minimum Data Standards & \\
\hline & General Queries & \\
\hline & Human Mutation Analysis Platform (HUMA) & \\
\hline & Job Management System (JMS) & \\
\hline & Node Accreditation & \\
\hline & NetCapDB & \\
\hline & Website/Mailing List & \\
\hline \multirow[t]{5}{*}{ Additional Support } & REDCap & Linux: Getting Started \\
\hline & Data Management & Linux: Configuring and securing your server \\
\hline & Software Programming & Install, configure and use Globus \\
\hline & Software License Request & Online to transfer large datasets \\
\hline & System Administration & Setup and configure a basic HPC cluster \\
\hline
\end{tabular}

queries can be divided into two broad categories, H3ABioNet-specific queries (e.g. node accreditation; NetCapDB; software license and data requests), and queries which deal with data analysis applications (e.g. workflow, scripting and software support).
H3ABioNet-specific queries tend to accumulate with the introduction of new activities and (or) tools in the network. This is exemplified in the network's introduction of the node accreditation project; a project which consists of exercises designed to assess the capacity of

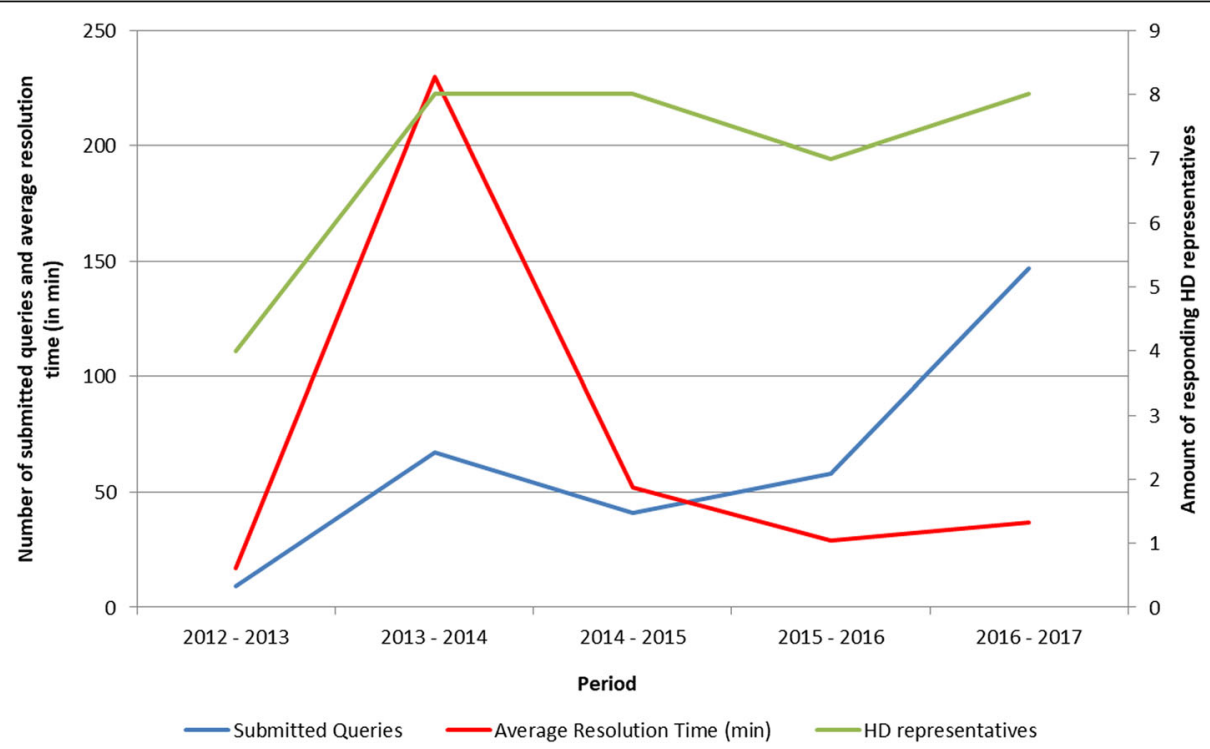

Fig. 1 Distribution of submitted queries, H3A-HD representatives and average resolution time from 1 August 2012 to 1 August 2017 


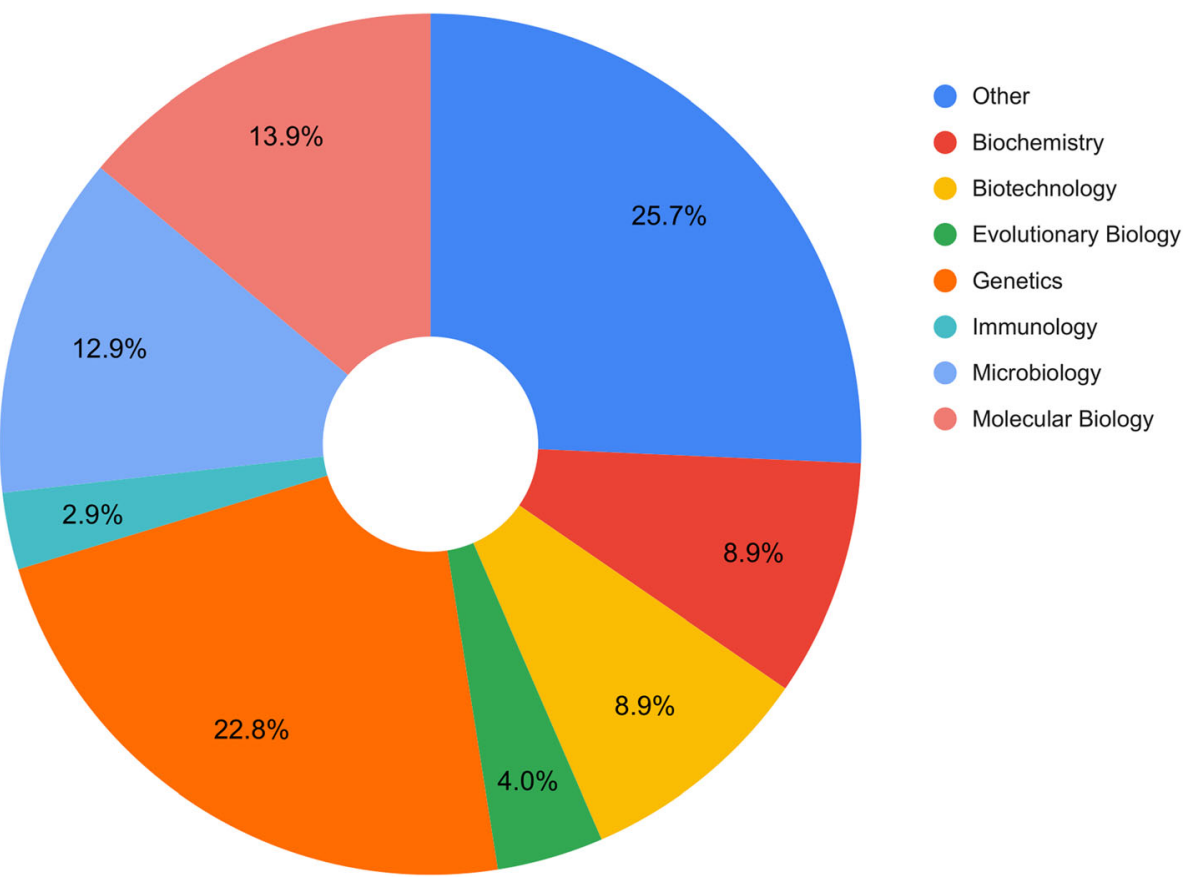

Fig. 2 Distribution of survey respondents' research fields

H3ABioNet nodes to properly process and analyse datasets typically produced by H3Africa. Network members typically use the Helpdesk to submit their intentions for node assessment and to obtain scripting or tool use support when conducting their assessments. Likewise, during H3ABioNet's infancy, several queries involved the setup and installation of servers, and similar trends followed the introduction of NetCapDB, a network reporting system, and various software courses.
Project-specific queries are typically received during the planning or data analysis of a given user's research project. Queries generated during project preparation include queries involving computing requirements, software inquiries, comparisons and recommendations. Software recommendations are generally within the scope of GWAS, WES and 16S data analysis. Queries received during data analysis involve the preparation of appropriate computing environments for data analysis,

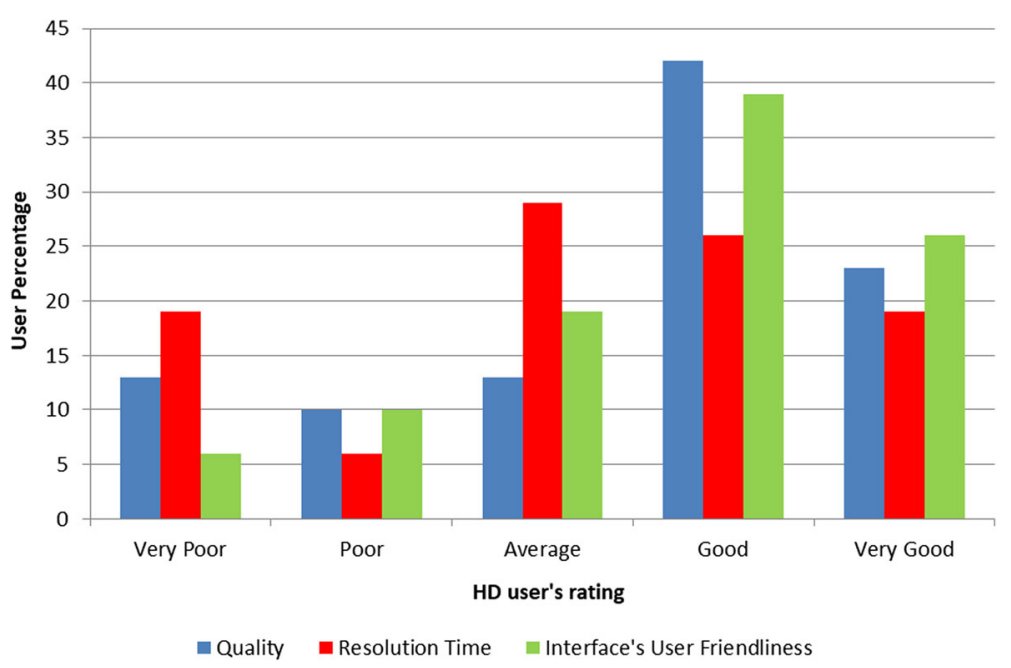

Fig. 3 H3A-HD users' evaluation of HD's quality, resolution time and user friendliness 


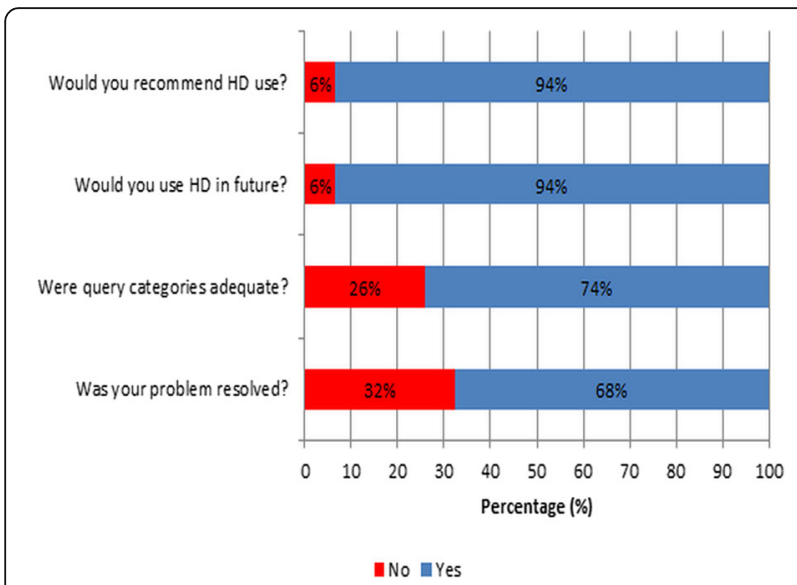

Fig. $4 \mathrm{H} 3 \mathrm{~A}-\mathrm{HD}$ user satisfaction with respect to various evaluation criteria

as well as scripting, particularly in relation to specific tools (e.g. FASTQ, Trimmomatic and BWA). Scripting languages may vary by query and queries also extend to the identification and resolution of errors during analysis.

\section{Discussion}

Due to several concerted initiatives, bioinformatics capacity in Africa is on the rise [12]. However, the demand still outweighs the supply, and researchers may often be left isolated, with little support, if their associated institutes are not equipped with dedicated support units [13]. The current report has introduced the H3ABioNet bioinformatics HD, a platform which can be employed to overcome such isolation. Although, the H3A-HD primarily serves African scientists, the service is available to all individuals seeking bioinformatics support. The H3AHD specifically serves the needs of African researchers because these concerns are not appropriately addressed by other support platforms. In addition, the HD representatives are uniquely aware and equipped to provide support for the bioinformatics challenges facing African researchers. Systematic integration of the H3A-HD into existing H3ABioNet activities and training events is essential to raising its awareness and to provide the learner community support following a training events [14].

Several lessons have been learned based on the initial implementation and evaluation of the H3A-HD which may be useful for the development and initiation of future HD iterations. Firstly, interactive communication methods are central to a HD's functionality and effectiveness. Ineffective communication mechanisms, query disputes and miscommunication are often the cause of unresolved queries. Effective and interactive communication can be achieved through increased email correspondence or live communication mechanisms such as video calling systems [15]. Secondly, ticketing strategies should be carefully developed prior to HD implementation, as the information acquired during ticket creation is essential to the maintenance and monitoring of an HD. The ticketing strategies employed may vary between HDs, and some HDs may employ more than one ticketing strategy. HDs commonly create tickets automatically via email correspondence, using Jira-based tracking [11] or case-based reasoning techniques [16]. Thirdly, the system used to implement the HD should be carefully evaluated based on the HD's aim. The H3A-HD makes use of a closed system because it may support research-specific queries, which may require privacy. Additionally, implementing the H3A-HD using a pre-existing and open source platform proved advantageous in terms of developmental work, however, using a supported platform may prove beneficial in the future, as relying on an unfunded, and opensource platform has the potential to compromise technical support and platform upgrading [17].

A number of key factors should be considered for future HD implementations. An HD should develop clear guidelines which outline the boundaries between query support and collaborative work. In the current report, assessment of user satisfaction is limited to a once-off, retrospective survey; however, best practice requires obtaining user feedback on a query-by-query basis. Such evaluation is crucial, and should be implemented in future H3A-HD iterations, as it provides real time evaluation, and may inform immediate alterations to the H3A-HD. A crucial limitation of the current evaluation is the omission of HD representatives. Reviews of an HD system should aim to assess the platform, the representatives, users and the management team to comprehensively identify key areas of success and improvement. Although a number of HDs exist within the bioinformatics and biological sciences community, associated publications are scarce, and there is a gap in helpdesk literature to compare to $[10,11,16]$. We hope to encourage others to publish their implementations, in order to build a knowledgebase for future implementations to build on.

To effectively sustain the H3A-HD, linkages with organizational bodies should be developed to sustain both financial and management support. Funding is an important component of the implementation, sustainability and success of an HD. Funded HDs generally have higher satisfaction rates than observed in the current report, however, the extent to which funding influences the quality of support provided by the H3A-HD and cannot be fully elucidated without evaluating the HD volunteers. Sustainability may require the production of a more comprehensive H3A$\mathrm{HD}$ resource through an increased number of $\mathrm{HD}$ representatives and (or) expansion of supported query categories. Additionally, support should be continuously updated and maintained by allowing common queries to guide SOP updates. 


\section{Conclusion}

H3ABioNet effectively launched one of the first free bioinformatics HDs in Africa, a flagship resource providing support to African researchers and beyond. Notably, communication mechanisms, ticketing strategies and usage frameworks are crucial to running a successful HD, and should be considered when developing or upgrading an HD. Because HDs are becoming crucial components of scientific research, more HDs need to make their design, management, usage and evaluation frameworks publically available, either online or through publication, to guide the development and implementation of future HDs. This will ensure that the gaps and strengths of existing HDs are easily identifiable and addressed, if necessary.

The H3A-HD has a proven track record of effective query management and user satisfaction. As a crucial component of H3ABioNet's support mechanism, the H3A-HD envisages becoming an important contributor to the effective increase of bioinformatics capacity in Africa. We encourage other HDs to make their development processes publicly available, as it may aid similar efforts in the effective development of their HDs.

\section{Methods}

To monitor progress and deliver improvement strategies, H3ABioNet frequently audits the H3A-HD using automatically generated logs accessible from the Joomla platform. The logs include query volume and resolution time. For this report, logs from the Joomla platform were analysed from August 2012 to August 2017.

A retrospective user satisfaction survey was created to assess user satisfaction with regards to support quality, resolution time and user friendliness, as well as future use and recommendation, from the users' perspective (see Additional file 1). The online and anonymous feedback survey was distributed to 244 participants to assess levels of user satisfaction. Data from the survey was collected and managed using REDCap (Research Electronic Data Capture) version 7.5.0, electronic data capture tools, hosted at The Centre for Proteomic and Genomic Research (CPGR).

Qualitative evaluation was also conducted based on the submitted queries to identify relevant themes with regards to the submitted queries and identify the types of applications in which African scientists require assistance.

\section{Supplementary information}

Supplementary information accompanies this paper at https://doi.org/10. 1186/s12859-019-3322-3.

Additional file 1. Usesr Satisfaction Survey

\section{Abbreviations}

H3ABioNet: H3Africa Bioinformatics Network; H3Africa: Human Heredity and Health of Africa Consortium; H3A-HD: H3ABioNet Helpdesk; HD: Helpdesk JMS: Job Management System; SOP: Standard Operating Procedure

\section{Acknowledgements}

The authors would like to acknowledge all H3A-HD personnel, including developers, representatives and administrators, particularly the Computational Biology Division, University of Cape Town, for their dedication and maintenance of the $\mathrm{H} 3 \mathrm{~A}-\mathrm{HD}$.

\section{Organization description}

$\mathrm{H} 3 \mathrm{ABioNet}$ is a pan-African bioinformatics network comprising 28 bioinformatics research groups distributed amongst 16 African countries and 1 partner institution in the USA. The consortium supports H3Africa researchers and their projects whilst developing bioinformatics capacity within Africa.

\begin{abstract}
Authors' contributions
JK, SP, SPS, JKK, MM, VN, and NM were crucial members of the development of the H3A-HD and play important roles as maintainers of the H3A-HD. JK and LZ led the writing of the manuscript, and VN, SPS, AO, AK. GW, NJM contributed significantly to specified sections and general editing of the final manuscript. All authors have read and approved the final manuscript
\end{abstract}

\section{Funding}

The H3ABioNet, is supported by the US National Institutes of Health Common Fund [grant numbers U41HG006941, U24HG006941]. The content of this publication is solely the responsibility of the authors and does not necessarily represent the official views of the National Institutes of Health. The funding body did not play any roles in the design of the study, collection, analysis, and interpretation of data and in writing the manuscript.

Availability of data and materials

The datasets used and/or analysed during the current study are available from the corresponding author on reasonable request.

Ethics approval and consent to participate

To conduct the user satisfaction survey, ethical clearance was received from the Research Ethics Committee of the Centre for Higher Education Development, University of Cape Town. Additionally, consent was ascertained through the completion of the user satisfaction survey.

Consent for publication

Not applicable.

\section{Competing interests}

The authors declare that they have no competing interests.

\section{Author details}

${ }^{1}$ Centre for Proteomic and Genomic Research, Cape Town, Western Cape, South Africa. ${ }^{2}$ Computational Biology Division, IDM, Faculty of Health Sciences, University of Cape Town, Cape Town, Western Cape, South Africa. ${ }^{3}$ Kumasi Centre for Collaborative Research in Tropical Medicine, Kwame Nkrumah University of Science and Technology, Kumasi, Ghana. ${ }^{4}$ Uganda Virus Research Institute, Entebbe, Uganda. ${ }^{5}$ Center for Informatics Science, Nile University, 6th October City, Egypt.

Received: 30 April 2019 Accepted: 16 December 2019 Published online: 30 December 2019

\section{References}

1. Schatz MC. Biological data sciences in genome research. Genome Res. 2015 Oct;25(10):1417-22.

2. Chimusa ER, Mbiyavanga M, Masilela V, Kumuthini J. "Broadband" Bioinformatics Skills Transfer with the Knowledge Transfer Programme (KTP): Educational Model for Upliftment and Sustainable Development. PLoS Comput Biol. 2015;11(11):e1004512.

3. Mulder NJ, Adebiyi E, Adebiyi M, Adeyemi S, Ahmed A, Ahmed R, et al. Development of bioinformatics infrastructure for genomics research. Glob Heart. 2017;12(2):91-8.

4. Mulder NJ, Adebiyi E, Alami R, Benkahla A, Brandful J, Doumbia S, et al. H3ABioNet, a sustainable pan-African bioinformatics network for human heredity and health in Africa. Genome Res. 2016;26(2):271-7.

5. Adoga MP, Fatumo SA, Agwale SM. H3Africa: a tipping point for a revolution in bioinformatics, genomics and health research in Africa. Source Code Biol Med. 2014;9:10. 
6. Gurwitz KT, Aron S, Panji S, Maslamoney S, Fernandes PL, Judge DP, et al. Designing a course model for distance-based online bioinformatics training in Africa: the H3ABioNet experience. PLoS Comput Biol. 2017;13(10):e1005715.

7. Fuller JC, Khoueiry P, Dinkel H, Forslund K, Stamatakis A, Barry J, et al. Biggest challenges in bioinformatics. EMBO Rep. 2013;14(4):302-4.

8. Tastan Bishop Ö, Adebiyi EF, Alzohairy AM, Everett D, Ghedira K, Ghouila A, et al. Bioinformatics education--perspectives and challenges out of Africa. Brief Bioinform. 2015 Mar;16(2):355-64.

9. Govindarajulu C. The status of helpdesk support. Commun ACM. 2002;45(1):97-100.

10. Yates A, Akanni W, Amode MR, Barrell D, Billis K, Carvalho-Silva D, et al. Ensembl 2016. Nucleic Acids Res. 2016:44(Database issue):D710-6.

11. Krishnakumar V, Kim M, Rosen BD, Karamycheva S, Bidwell SL, Tang H, et al. MTGD: the Medicago truncatula genome database. Plant Cell Physiol. 2015 Jan;56(1):e1.

12. H3Africa Consortium, Rotimi C, Abayomi A, Abimiku A, Adabayeri VM, Adebamowo C, et al. Research capacity. Enabling the genomic revolution in Africa. Science. 2014;344(6190):1346-8.

13. Karikari TK. Bioinformatics in Africa: the rise of Ghana? PLoS Comput Biol. 2015;11(9):e1004308

14. Searls DB. Ten simple rules for online learning. PLoS Comput Biol. 2012 Sep 13:8(9):e1002631.

15. Garcia G, Bertoia A, Cameselle L, Benitez S, Giunta D, Baum A, et al. Communication problems between end-users and technicians through a help desk in a health information system. Stud Health Technol Inform. 2015;216:985.

16. Chang KH, Raman P, Carlisle WH, Cross JH. A self-improving helpdesk service system using case-based reasoning techniques. Comput Ind. 1996; 30(2):113-25.

17. Vincent AT, Charette SJ. Freedom in bioinformatics. Front Genet. 2014;5 Available from: https://www.ncbinlm.nih.gov/pmc/articles/PMC4116781/.

\section{Publisher's Note}

Springer Nature remains neutral with regard to jurisdictional claims in published maps and institutional affiliations.

Ready to submit your research? Choose BMC and benefit from:

- fast, convenient online submission

- thorough peer review by experienced researchers in your field

- rapid publication on acceptance

- support for research data, including large and complex data types

- gold Open Access which fosters wider collaboration and increased citations

- maximum visibility for your research: over $100 \mathrm{M}$ website views per year

At $\mathrm{BMC}$, research is always in progress.

Learn more biomedcentral.com/submissions 